\title{
Total Transcatheter Stage 1: A Word of Caution
}

\author{
Stephen Nageotte ${ }^{1}\left[\right.$ [ $\cdot$ Shabana Shahanavaz ${ }^{2} \cdot$ Pirooz Eghtesady $^{3} \cdot$ David Balzer $^{1}$
}

Received: 15 February 2021 / Accepted: 21 April 2021 / Published online: 29 April 2021

(c) The Author(s), under exclusive licence to Springer Science+Business Media, LLC, part of Springer Nature 2021

\begin{abstract}
For patients with single ventricle physiology, being able to initially establish systemic blood flow and control pulmonary blood flow is critical to their long-term health. Recently, there have been descriptions in achieving this by a purely transcatheter approach with stenting of the ductus arteriosus and implanting pulmonary flow restrictors, a very appealing prospect. We review a case series of 6 patients who underwent a percutaneous modified stage 1 approach using modified Microvascular plugs (MVP) at our center between September 2019 and December 2019. The initial procedure was technically successful in all patients with single-stage ductal stenting and placement of bilateral modified MVP via femoral access. Four patients underwent repeat cardiac catheterization prior to subsequent surgery that demonstrated elevated Qp:Qs (>2:1) in 3 of the 4 patients with an elevated mean distal PA pressure $>20 \mathrm{mmHg}$ in all patients. In some patients, the device migrated into the distal right pulmonary artery. One patient after Glenn shunt was found to have significant LPA stenosis requiring stenting. While the percutaneous modified stage 1 approach is a promising approach, we offer a word of caution against widespread adoption of this technique with the currently available devices.
\end{abstract}

Keywords Single ventricle $\cdot$ Flow restrictor $\cdot$ Percutaneous modified stage 1

\section{Introduction}

Schranz et al. [1] recently published a research letter entitled "Hypoplastic Left Heart Stage 1: No Norwood, No Hybrid" in which they describe performing a first stage single ventricle palliation by a purely transcatheter approach. This is a very appealing idea that was first reported in 2001 using modified Amplatzer devices [2,3]. This concept can potentially be applied to any lesion in which control of pulmonary blood flow is needed. Following the report by Khan et al. [4] using modified Microvascular plugs (MVP) (Medtronic Inc., Minneapolis, MN) as flow restrictors, our group also applied

Stephen Nageotte

Snageotte@wustl.edu

1 Division of Cardiology, Department of Pediatrics, Washington University in St. Louis School of Medicine, One Children's Place, Northwest Tower, Box 8116, St. Louis, MO 63110, USA

2 Cincinnati Children's Hospital Medical Center, The Heart Institute, University of Cincinnati College of Medicine, Cincinnati, OH, USA

3 Department of Surgery, Department of Pediatrics, Washington University in St. Louis School of Medicine, St. Louis, MO, USA this technique to a small series of patients and we would like to report our case series as a word of caution prior to widespread application of this technology.

\section{Case Series}

A total of six patients underwent a percutaneous modified stage 1 approach at our center between September 2019 and December 2019 (Table 1). All patients were discussed at multidisciplinary conference prior to the procedure. Patients in the initial part of the experience were deemed higher risk surgical candidates or needed time to decision of single ventricle vs. biventricular repair. All our patients underwent single-stage ductal stenting with placement of bilateral MVP via femoral access. Patients with restriction at the atrial level underwent intervention on the atrial septum. Two patients underwent both a static balloon dilation across the atrial septum as well as Rashkind atrial septostomy at the time of the percutaneous modified stage 1 . One patient with hypoplastic left heart syndrome, intact atrial septum and total anomalous pulmonary venous return initially underwent stenting of an obstructed vertical vein immediately after birth followed by 


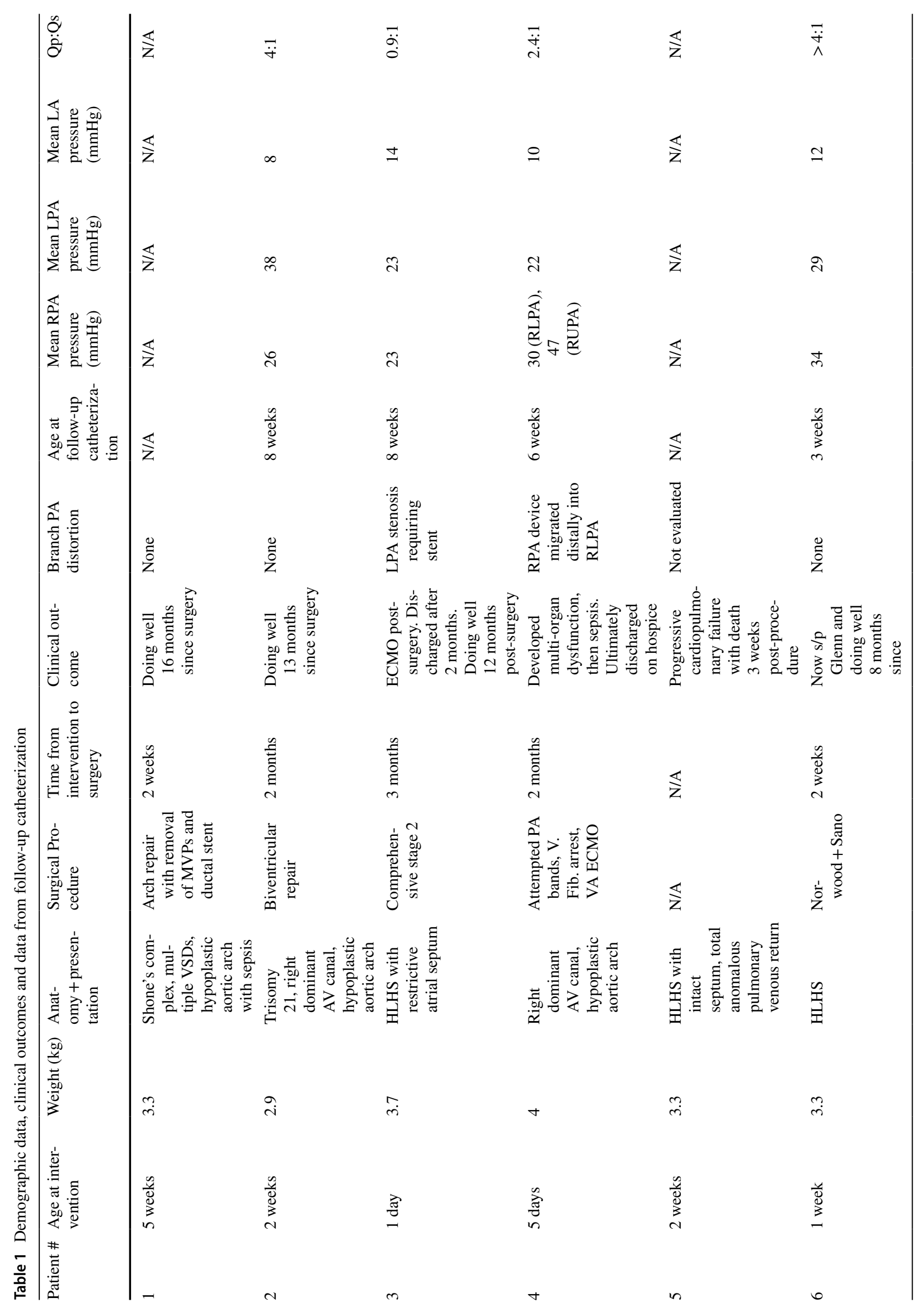


stenting of the atrial septum at 3 days of life, with percutaneous modified stage 1 at 2 weeks of life.

The modification of the MVPs for the initial case was performed by excision of the Polytetrafluoroethylene (PTFE) membrane using a scalpel as described previously [1, 4]. This presented some challenges due to difficulty in stabilizing a soft device and accurately creating consistently sized defects. After the initial case, we utilized a low-temperature fine tip Eye Bovie cautery (Symmetry Surgical, Antioch, TN) to modify the MVP and create an opening that measured 3-4 mm (Fig. 1). To create a symmetrical opening, the fenestration was also dilated with a $3 \mathrm{~mm}$ balloon in patient 4.

Implantation of 5Q and 7Q MVPs was performed with four Fr Judkins Right two catheters (Cordis, Santa Clara, California). The $9 \mathrm{~mm}$ MVP was deployed via a Flexor ${ }^{\circledR}$ Check-Flo® sheath (Cook Medical, Bloomington, IN). The Right Pulmonary Artery (RPA) device was harder to position secondary to challenges with maintaining a stable catheter position in an acutely angled RPA in infants.

One patient had an acute desaturation immediately after RPA device implantation that warranted replacing the device with a larger fenestration. Final angiography demonstrated that the RPA device migrated distally in five out of six patients after they were released and crossed the upper lobe takeoff (Fig. 2). This was despite oversizing the device, using the $7 \mathrm{Q}$ device in vessels that measured 3.7-5.7 $\mathrm{mm}$ and the $9 \mathrm{Q}$ device in a vessel measuring $7.6 \mathrm{~mm}$. Regardless of this, there remained good flow into all branches of each pulmonary artery and so the devices were left in place. There were no procedural complications.

Procedural details and outcomes are provided in Table 1. The patients were monitored in the cardiac Intensive Care Unit post-procedure and did not have any immediate management challenges. All the patients but one had saturations in the high 80 s in the immediate post-procedure period with echo Doppler demonstrating a restrictive pattern with diastolic continuation in branch pulmonary arteries. One patient underwent a repeat catheterization for concern of overcirculation where the initial devices were removed and larger devices with smaller fenestrations were placed to provide more restriction to pulmonary blood flow. Despite this, the patient remained overcirculated and was taken to the OR for a Norwood procedure several days later. The patient with hypoplastic left heart syndrome, intact atrial septum and total anomalous pulmonary venous return referenced earlier had progressive
Fig. 1 Modified Medtronic Microvascular Plug (MVP). a The inflow portion of the PTFE on the device is being modified using a low-temperature fine tip Eye Bovie cautery (Symmetry Surgical, Antioch, TN) prior to loading the device into the catheter. b The fenestration is being ballooned using a $3 \mathrm{~mm}$ Maverick TM PTCA balloon (Boston Scientific, Marlborough, MA). c A fenestration has been created in the inflow portion of the device. $\mathbf{d}$ Another angle further demonstrates the fenestration

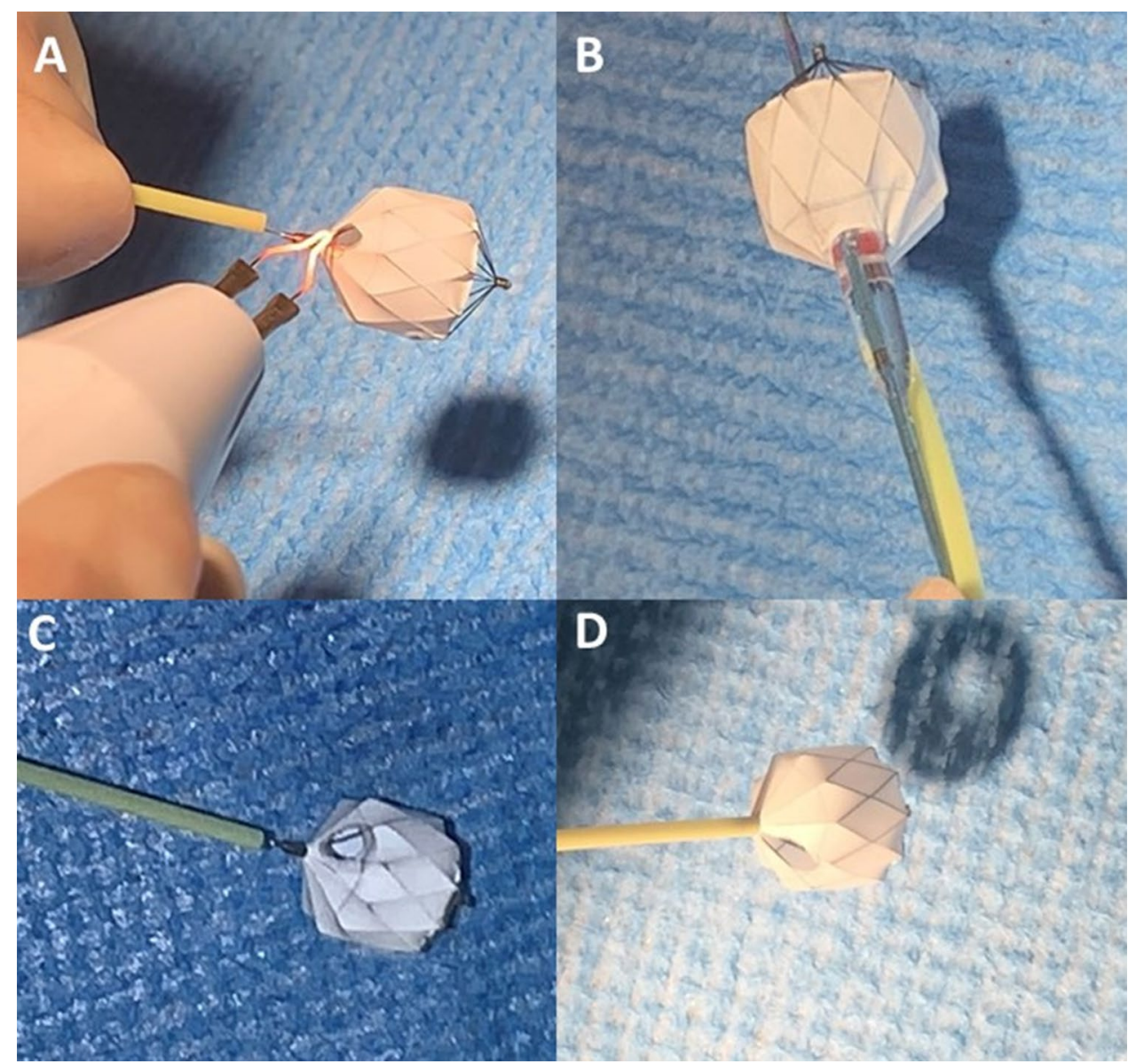




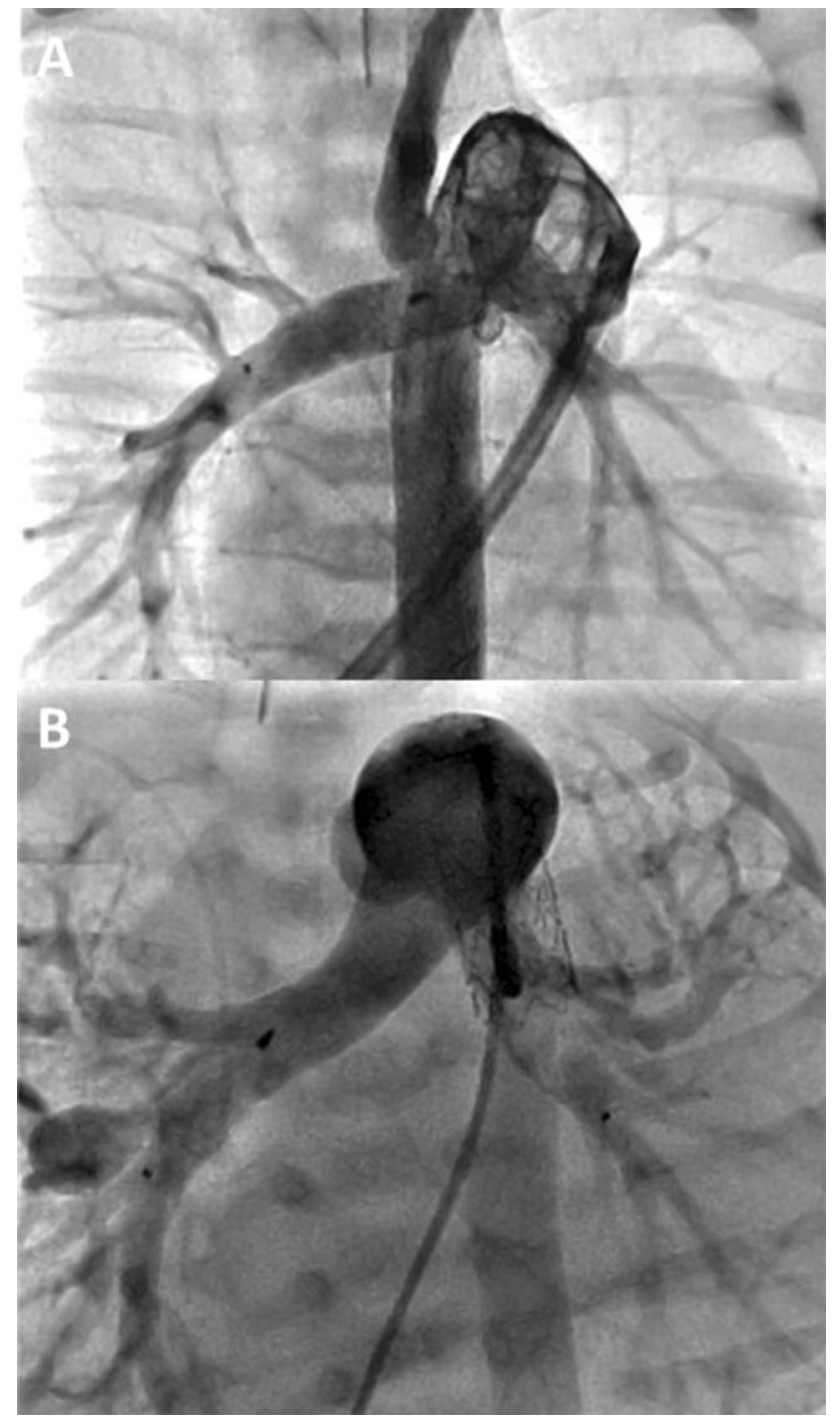

Fig. 2 Angiographic evaluation of the modified devices. a Final angiogram post modified MVP placement in the RPA. The single arrows demonstrate the proximal and distal markers of the MVP and the double arrow depicts the takeoff of the right upper pulmonary artery. b Angiographic evaluation of the modified devices 1 month post initial procedure shows distal migration of the RPA device into the RLPA with an unprotected RUPA, demonstrated by the double arrow

deterioration with evidence of significant pulmonary disease, declining saturations, poor ventilator mechanics and ultimate death at 1 month of age.

Four patients underwent repeat cardiac catheterization prior to subsequent surgery that demonstrated elevated Qp:Qs (>2:1) in three of the four patients with an elevated mean PA pressures $>20 \mathrm{~mm} \mathrm{Hg}$ in all patients. Qp:Qs was $\geq 4: 1$ in two of the four patients. Pulmonary vascular resistance could not be accurately calculated since we did not routinely perform perfusion scans and the mean pulmonary artery pressures varied somewhat between lungs. One patient struggled with pulmonary blood flow immediately after Glenn surgery and was found to have Left Pulmonary Artery (LPA) stenosis after device removal necessitating implantation of a stent.

\section{Discussion}

Total percutaneous stage 1 palliation is a noble goal predicated upon the ability to provide unobstructed systemic blood flow (ductal stenting), unobstructed pulmonary venous return (atrial septostomy or stenting) and controlled pulmonary blood flow. It is this latter issue which has remained a challenge. Following the animal work done by Khan et al. [4] on the use of modified MVP, this technique was applied to humans by the group in Giessen, Germany as well as our group. The report by Schranz et al. [1] demonstrated excellent outcomes in a group of 6 neonates, although, similar to our experience, one of their patients needed removal and replacement of the MVPs secondary to pulmonary overcirculation and a second needed LPA stenting. No follow-up catheterization data was provided. We report our experience to provide a word of caution prior to any widespread implementation of this technique.

The modified MVP is an attractive device to be used as an internal band due to its low profile and easy retrievability, but there are several limitations that need to be overcome before these devices can be used widely. First, creating the fenestration in the MVPs with a scalpel can be difficult. We found that the use of the eye Bovie greatly facilitated creation of a fairly uniform circular fenestration in the PTFE. Appropriate sizing can then be done by further dilation of the hole with a $3 \mathrm{~mm}$ coronary balloon (Fig. 1). The bigger challenge was the inability to ascertain the appropriate sized fenestration. The ideal internal band would balance circulations and protect pulmonary vasculature for single ventricle palliation. While we initially used the approach previously described in the literature [1,4], we later made smaller fenestrations with the eye Bovie. Despite adjusting our techniques of fenestration creation and size, several of our patients continued to demonstrate evidence of pulmonary overcirculation with elevated distal pulmonary artery pressures. This was despite appropriate device sizing as confirmed angiographically and limitation of flow through the fenestration as confirmed by Doppler. We believe that some of this could be attributed to potential flow around the device. In patient 4 we attempted to cross the fenestration that we created in the modified MVPs with a coronary guidewire and balloon. Although technically very easy to achieve, we were unable to confirm that the wire and balloon had traversed the fenestration or whether we were alongside the device. The fact that we crossed so easily suggested that we were alongside the MVP rather than through the 
fenestration. However, the possibility remains that despite making the fenestrations even smaller, they remained too large. Some of the Hybrid literature suggests making the PA bands closer to $2.5 \mathrm{~mm}$ in luminal diameter as a target [5]] and perhaps that should have been the ideal fenestration size we aimed for.

In our experience, the devices did have a tendency to migrate distally especially in the RPA (Fig. 2). This can both result in jailing of the distal branches and subject the upper lobe arteries to high pressure. The device relies on the tension created by the outward expansion of the nitinol against the vessel wall which can be overcome by the systemic pressure behind the device especially in a pulsatile PA. The presence of the ductal stent in close proximity to the LPA device appear to provide more stability preventing distal displacement. In addition, another reason for the continued overcirculation may be because the restriction is only at the level of a very thin membrane which may not impose enough resistance to flow when subjected to systemic pressures.

These devices are very easy to remove both via transcatheter technique and surgically when they have been in place for a few weeks. However, as noted by Khan et al., only 50\% of devices can be removed by snares 12 weeks following the procedure. Patient 3 in our series had the devices in place for 96 days and following surgical removal which, although not technically challenging, caused LPA narrowing necessitating stent placement (Fig. 3). This appears similar to the patient reported by Schranz et al. although the time period the device was in place was not specified in their report. This has obvious implications for any single ventricle patient. Branch pulmonary artery stenosis has been a drawback of the hybrid approach as well and is something that needs to be considered in strategizing the management of these patients.

In our experience, the modified Medtronic Vascular Plug is not a perfect device to act as a pulmonary flow restrictor. The ideal device would be easily implantable and retrievable without disruption of the vessel wall, would have enough radial strength to prevent migration into the distal vessel and, perhaps most importantly, would have an easily modifiable mechanism to regulate pulmonary blood flow. There needs to be no flow around the outside of the device once in place.

\section{Conclusion}

We applaud the pursuit of totally percutaneous stage 1 palliation as detailed in the report of Schranz et al. and we feel that this work should continue. However, we offer the above word of caution regarding currently available devices. The application of this technique is yet to be refined. We acknowledge that as in any newly emerging procedure, there

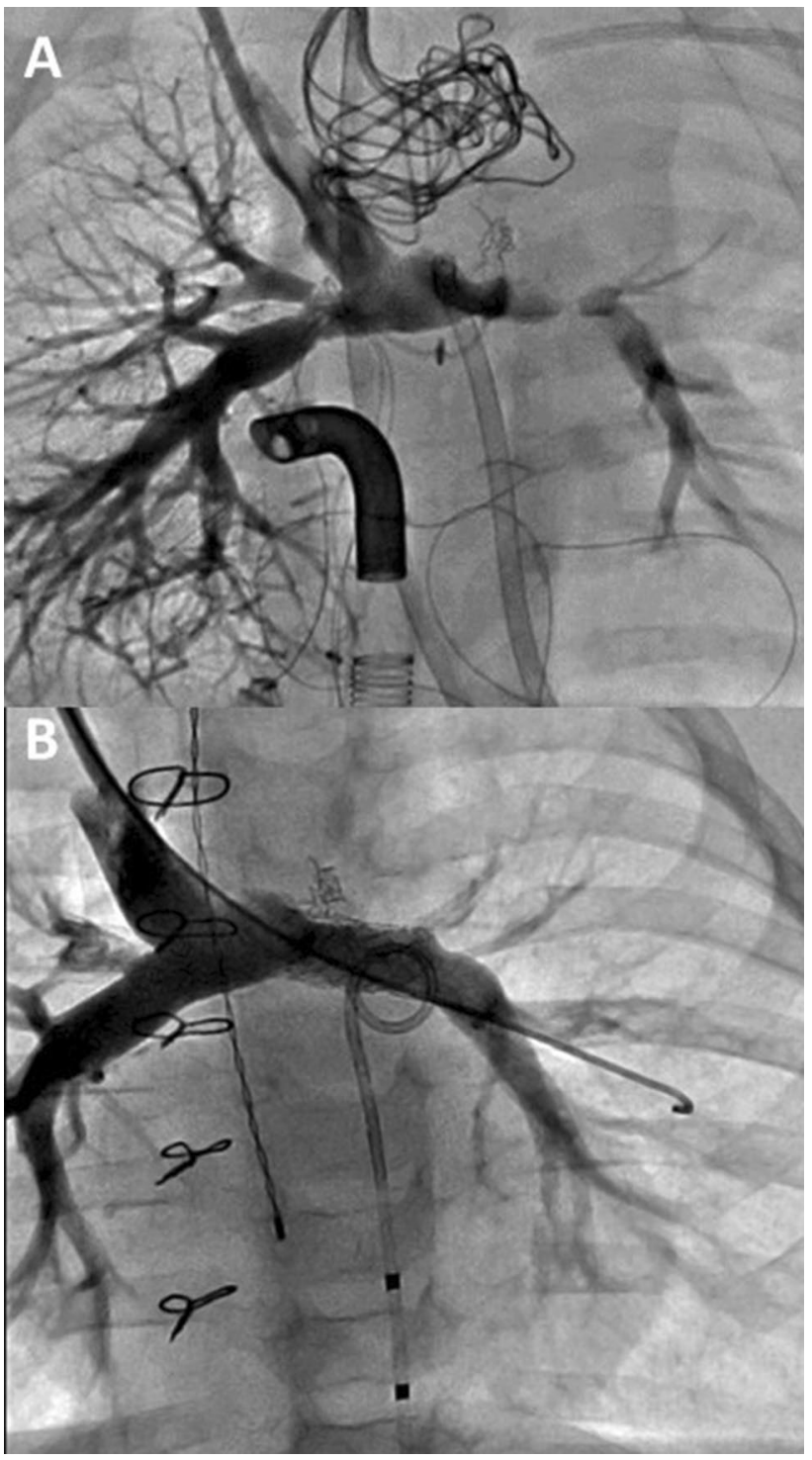

Fig. 3 Angiographic evaluation of the Pulmonary Arteries post comprehensive stage 2. a Angiography performed in the SVC post comprehensive stage 2 shows mild distortion of the RPA in the region of the device. There is moderate stenosis of the LPA at the bifurcation which corresponds to the position of distal end of the modified MVP device. b Follow-up angiography demonstrates improvement in vessel caliber post-LPA stent with a $7 \mathrm{~mm} \times 17 \mathrm{~mm}$ Valeo $^{\mathrm{TM}}$ Balloon Expandable Biliary Stent (B Bard, Tempe, AZ)

is a steep learning curve prior to success. This procedure is perhaps appropriate with the currently available technology as a short-term palliation in two ventricle patients with excessive pulmonary blood flow prior to complete repair. However, we would encourage caution in our single ventricle population in whom tightly regulating pulmonary blood flow is critical to their future success. Finally, we strongly encourage collaboration between individual and industry investigators to develop flow restrictor devices that will overcome the limitations detailed in this report. 


\section{Declarations}

Conflict of interest Drs. Balzer and Shahanavaz are proctors for Medtronic, Inc.

\section{References}

1. Schranz D, Esmaeili A, Schrewe R et al (2020) Hypoplastic left heart stage I: no norwood, no hybrid. Circulation 142:1402-1404. https://doi.org/10.1161/CIRCULATIONAHA.120.047668

2. Chan KC, Mashburn C, Boucek MM (2006) Initial transcatheter palliation of hypoplastic left heart syndrome. Catheter Cardiovasc Interv 68:719-726. https://doi.org/10.1002/ccd.20669

3. Chan KC, Gleason BK, Bright JM, Boucek MM (2001) Percutaneous Implantable and Retrievable Pulmonary Arterial Band: Early animal experience. 3 rd World Congress of Pediatric Cardiology \& Cardiac Surgery: Toronto, Ontario, Canada, 27-31 May, 2001. Cardiol Young 11:274. https://doi.org/10.1017/S10479511000121 42

4. Khan AH, Hoskoppal D, Kumar TKS et al (2019) Utility of the medtronic microvascular plug $^{\mathrm{TM}}$ as a transcatheter implantable and explantable pulmonary artery flow restrictor in a swine model. Catheter Cardiovasc Interv 93:1320-1328. https://doi.org/10. $1002 / \mathrm{ccd} .28162$

5. Davies RR, Radtke WA, Klenk D, Pizarro C (2014) Bilateral pulmonary arterial banding results in an increased need for subsequent pulmonary artery interventions. J Thorac Cardiovasc Surg 147:706-712. https://doi.org/10.1016/j.jtcvs.2013.10.038

Publisher's Note Springer Nature remains neutral with regard to jurisdictional claims in published maps and institutional affiliations. 